\title{
Sewer inspection and comparison of acoustic and CCTV methods
}

1 Anna Romanova BEng

PhD Student, School of Engineering Design and Technology, University of Bradford, Bradford, UK

2. Kirill V. Horoshenkov BEng, PhD

Professor of Acoustics, School of Engineering Design and Technology, University of Bradford, Bradford, UK
3 Simon J. Tait BEng, PhD

Professor of Civil Engineering, School of Engineering Design and Technology, University of Bradford, Bradford, UK

4 Thomas Ertl BEng, PhD

Professor of Wastewater and Water Pollution Control, Department of Water, Atmosphere and Environment, Institute for Sanitary Engineering and Water Pollution Control, University of Natural Resources and Applied Life Sciences, Vienna, Austria
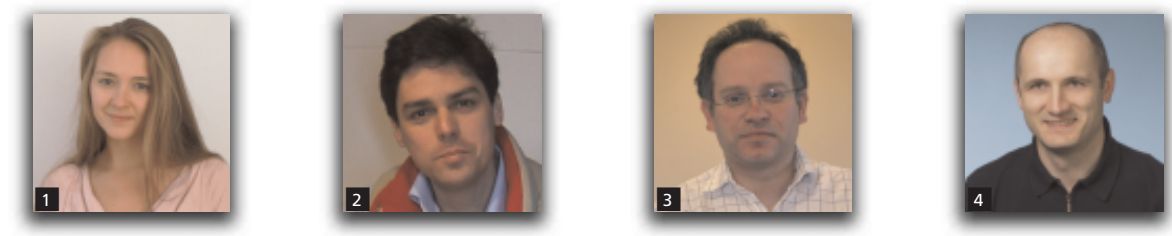

In the UK, the majority of sewer pipe inspections are carried out using closed-circuit television (CCTV) technology. This inspection technology is expensive; given the size of the UK sewer network, this means that only a small sample of network is inspected regularly. Up-to-date condition information on individual sewers is required to minimise sewer network operation failures and, ultimately, to eliminate flooding. There is therefore a need for a quicker and cheaper inspection method. This paper reports on a novel low-cost acoustic sensor system that can be used for the rapid detection of various defects in sewer pipes. It is shown that a large number of pipe defects can be classified and validated against CCTV images by way of visual examination of the acoustical data presented in the form of spectrograms. An overview of the technological principles used by the acoustic inspection method is presented in this paper together with the results of field trial surveys. The new method was tested in operational sewers in Austria and the acoustic inspection results compared with available CCTV reports: $79 \%$ of the defects identified by CCTV were also detected using the acoustic technique.

\section{Introduction}

In many countries, wastewater collection and drainage in urban areas is carried out using an extensive system of underground pipes. The UK drainage system has developed over many years and there are now over $300000 \mathrm{~km}$ of pipes. These assets have to be monitored and maintained in order to deliver the desired quality of service to the community. Failure to maintain a sewer pipe in an acceptable condition may result in asset failures such as structural collapse or a blockage that may cause operational performance problems.

The most commonly used sewer pipe inspection technique is by closed-circuit television (CCTV). This technology was first introduced in the late 1960s and has dominated the sewer pipe inspection market since then. CCTV technology, which is remotely controlled, uses a manually pushed or motorised trolley with a camera to capture a series of images. These images are then visually assessed by an operator to identify individual defects and estimate the condition inside the pipe using standard methods of classification.
The advantages of the method are that it provides a series of images of the pipe wall that can be stored on site and then examined later. During subsequent examination of the collected images, any defect can be observed, its location noted and the type of defect classified based on a standard method of classification (e.g. WRC, 2004).

Based on the information obtained from CCTV studies, standard defect classification systems were created over time. Among these are the classification system used in the UK (WRC, 2004) and the European classification system (CEN, 2002) adopted in most other EU countries. Defect classification systems are similar in concept where they provide a coding system that classifies the defect and its condition, orientation, scale, and the estimated effect on the inspected pipe's general operational performance. All of this information is stored in an assigned number-letter code. Once the number and scale of the defects and their locations inside the pipe are known, this information is reported to the sewer network operator in a standard format. This 